\title{
Point-of-care monitoring of perioperative intraocular pressure using portable tonometry in a patient with Posner-Schlossman syndrome
}

\section{-a case report-}

\author{
Sung-Hoon Kim ${ }^{1}$, Jin-Ho Rhim ${ }^{1}$, Young-Jin Moon ${ }^{1}$, Jihion Yu ${ }^{1}$, Jong-Yeon Park ${ }^{1}$, and \\ Ashish Bangaari ${ }^{2}$ \\ ${ }^{1}$ Department of Anesthesiology and Pain Medicine, Asan Medical Center, University of Ulsan College of Medicine, Seoul, Korea, \\ ${ }^{2}$ Department of Anaesthesia, Intensive Care and Pain Management, Axon Anaesthesia Associates, Global Hospitals, Hyderabad, India
}

A 56-year-old man with a rotator cuff injury, scheduled for arthroscopic reconstruction surgery, had a history of recurrent symptoms of eyeball pain and blurred vision for several years. After close examination, he was diagnosed with PosnerSchlossman syndrome. Three weeks before the scheduled surgery, his intraocular pressure (IOP) increased (> $30 \mathrm{mmHg}$ ) and he became extremely anxious about the surgery. We monitored his IOP intraoperatively and successfully completed general anesthesia without any sequelae. As Posner-Schlossman syndrome can present with severe complications that may lead to postoperative visual loss, intraoperative monitoring of intraocular monitoring and careful anesthetic management are crucial to protect vision. (Korean J Anesthesiol 2014; 66: 248-251)

Key Words: Anesthesia, Intraocular pressure, Monitoring, Posner-Schlossman syndrome, Tonometry.

Posner-Schlossman syndrome is a rare inflammatory ocular disease that presents as recurrent episodes of markedly increased intraocular pressure (IOP) and related symptoms. Although the symptoms of Posner-Schlossman syndrome are self-limiting and IOP usually normalizes in intervals between acute attacks, IOP can increase to above $40 \mathrm{mmHg}$ and may lead to severe complications, such as primary open-angle glaucoma or ischemic optic disc change [1]. During non-ophthalmic surgery under general anesthesia, patients with Posner-Schlossman syndrome may experience a sudden increase in IOP, and critical interruption of optic blood flow autoregulation can lead to postoperative vision loss [1,2]. With point-of-care monitoring of IOP, a 56-year-old man diagnosed with Posner-Schlossman syndrome successfully underwent arthroscopic rotator cuff repair under general anes-

Received: January 29, 2013. Revised: 1st, March 7, 2013; 2nd, March 20, 2013. Accepted: March 26, 2013.

Corresponding author: Sung-Hoon Kim, M.D., Department of Anesthesiology and Pain Medicine, Asan Medical Center, University of Ulsan College of Medicine, 388-1, Pungnap 2-dong, Songpa-gu, Seoul 138-736, Korea. Tel: 82-2-3010-3868, Fax: 82-2-3010-6790, E-mail: shkimans@ amc.seoul.kr

(c) This is an open-access article distributed under the terms of the Creative Commons Attribution Non-Commercial License (http:// creativecommons.org/licenses/by-nc/3.0/), which permits unrestricted non-commercial use, distribution, and reproduction in any medium, provided the original work is properly cited. 
thesia. We describe this patient and review the literature on anesthetic requirements and plans for IOP control in patients with this condition.

\section{Case Report}

A 56-year-old male, $168 \mathrm{~cm}$ tall and weighing $71 \mathrm{~kg}$, with a traumatic right rotator cuff injury, was scheduled for arthroscopic reconstruction surgery under general anesthesia. This patient had a history of diabetes mellitus and was taking oral hypoglycemic agents and insulin. For several years, he experienced recurrent episodes of severe left ocular pain and increased IOP, which later resolved. On close ophthalmic examination, he was diagnosed with Posner-Schlossman syndrome. There was mild epithelial edema of the cornea with a few keratic precipitates, and his cup/disc ratio was 0.5 with no visual field change. During the acute crisis period, the measured IOP in his left eye was above $30 \mathrm{mmHg}$ and the IOP in his right eye was $13 \mathrm{mmHg}$. His symptoms were relieved after applying eye drops containing dorzolamide, a carbonic anhydrase inhibitor, and prednisolone acetate, but frequently recurred. Three weeks before his scheduled surgery, the IOP in his left eye rose again and he became extremely anxious about the operation. After consultation with an ophthalmologist, we decided to measure the patient's IOP intraoperatively using portable tonometry, and obtained informed consent from the patient for this perioperative risk.

Preoperative laboratory findings, including hemoglobin concentration, liver function and coagulation tests, and arterial blood gas analysis, were normal, as were chest X-rays. The patient had a left axis deviation and an incomplete right fascicular block on electrocardiography and a mild restrictive pattern on

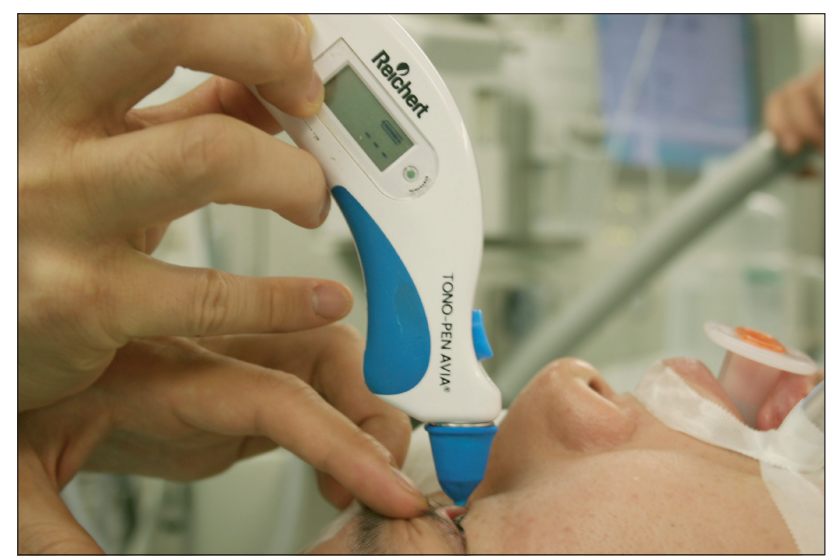

Fig. 1. Bedside measurement of intraocular pressure in the anesthetized patient using a portable tonometer. The transducer is gently tapped on the patient's cornea perpendicularly and pressure measured ten times. The device averages these readings and displays intraocular pressure on the screen. pulmonary function test without any significant symptoms. In the morning of the day of surgery, the patient received $7.5 \mathrm{mg}$ of midazolam per os. Before entering the operating room, his vital signs were stable, with a blood pressure of $133 / 75 \mathrm{mmHg}$, a heart rate of 82 beats per min, and an oxygen saturation of $99 \%$. Two drops of $0.5 \%$ proparacaine hydrochloride were applied to each eye, and IOP was measured by portable tonometry (Tono-Pen Avia, Ametek, NY, USA) (Fig. 1). Initially, IOP was 19 mmHg in his left eye and $16 \mathrm{mmHg}$ in his right eye. A 20-gauge catheter was inserted into his left radial artery to monitor continuous arterial pressure. Anesthesia was induced with propofol $100 \mathrm{mg}$, lidocaine $75 \mathrm{mg}$, rocuronium $50 \mathrm{mg}$, and a supplemental dose of fentanyl $100 \mu \mathrm{g}$, and ventilation was controlled to maintain end tidal carbon dioxide at $35 \pm 5 \mathrm{mmHg}$. Anesthesia was maintained with $2-2.5 \%$ sevoflurane in an oxygen-air mixture $\left(\mathrm{FiO}_{2}\right.$ $0.5)$. Immediately after tracheal intubation, IOP increased to $23 \mathrm{mmHg}$ in his left eye and $21 \mathrm{mmHg}$ in his right eye, but decreased within $5 \mathrm{~min}$ to $14 \mathrm{mmHg}$ and $12 \mathrm{mmHg}$, respectively. Initial arterial blood gas analysis showed an Hct of 39\%, a pH of 7.48, a $\mathrm{PaCO}_{2}$ of $34 \mathrm{mmHg}$, and $\mathrm{PaO}_{2}$ of $240 \mathrm{mmHg}$. After changing the patient's position to left lateral decubitus, the IOP was $15 \mathrm{mmHg}$ in his left eye and $14 \mathrm{mmHg}$ in his right side, but increased to $20 \mathrm{mmHg}$ and $14 \mathrm{mmHg}$, respectively, after skin incision and arthroscopic insertion. The concentration of inhaled sevoflurane was adjusted in response to instantaneous IOP variation, and the occurrence of IOP surge was closely monitored. During the 210 min operation, the patient's vital signs remained stable and no adverse event occurred. Serial measurements by portable tonometry showed that his IOP remained within an acceptable range throughout the entire perioperative period (Fig. 2). The patient's postoperative course was uneventful.

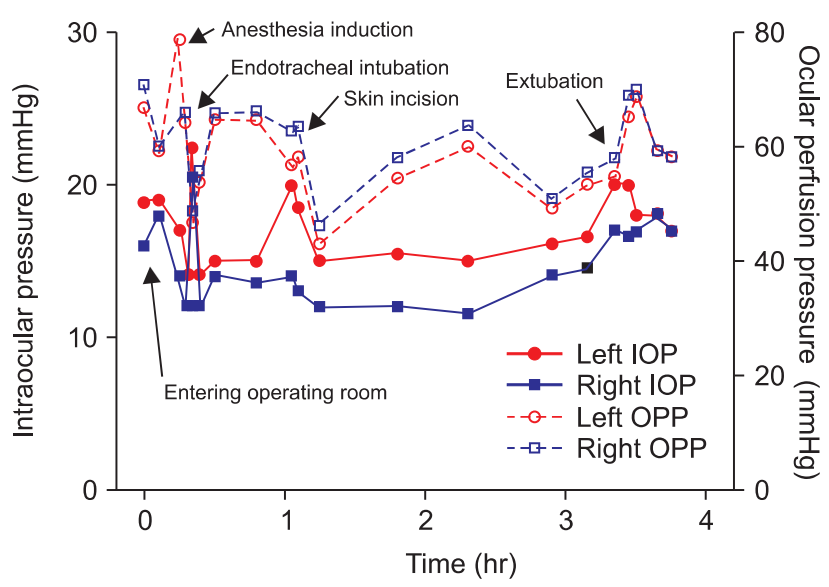

Fig. 2. Serial monitoring of perioperative intraocular pressure and ocular perfusion pressure by portable tonometry. IOP: intraocular pressure, OPP: ocular perfusion pressure. 


\section{Discussion}

Posner-Schlossman syndrome is an uncommon ocular hypertensive disorder, with a mean annual incidence of 0.4 per 100,000 population and a mean annual prevalence of 1.9 per 100,000 [1]. Its symptoms include blurred vision and/or ocular pain, which frequently subside in days to weeks. Moreover, Posner-Schlossman syndrome is associated with anterior uveitis and usually recurs periodically. Although its pathogenic mechanism is unclear, it has been associated with herpes simplex virus, cytomegalovirus infection, and autonomic dysfunction, and has been suggested as a variation of developmental glaucoma. Posner-Schlossman syndrome may be related to primary open-angle glaucoma and non-arteritic anterior optic neuropathy, which cause ischemic optic disc changes $[1,3]$. Because an acute increase in IOP, to over $40 \mathrm{mmHg}$ (normal 10-21 $\mathrm{mmHg}$ ), may be present for several days prior to the manifestations of symptoms, control of IOP during this period is critical $[3,4]$. Any potential interruption to optic blood flow autoregulation may lead to postoperative visual loss [2]. Partial or total blindness after non-ophthalmic surgery has been reported in patients who experience an increase in IOP beyond the normal range [5-7]. Although our patient was in a relapsed phase of Posner-Schlossman syndrome and his IOP was within the normal range at the time of surgery, attention is warranted during surgery because some of these patients have atypical presentation, with elevated IOP between attacks $[1,4]$.

Most anesthetic drugs and opioids lower IOP, and intravenous anesthetics such as propofol, thiopental, and etomidate reduce IOP surge $[3,8]$. Propofol, administered in a continuous intravenous manner, reduces IOP regardless of changes in blood pressure and heart rate [8]. Propofol seems to be a better anesthetic choice in these patients, as it protects against IOP, even in patients in the Trendelenburg position, possibly due to a sympatholytic effect [9]. Over the relatively short period of most operations, changes in IOP in patients anesthetized with propofol and sevoflurane did not differ significantly [10]. Vecuronium bromide, at a concentration of $0.1 \mathrm{mg} / \mathrm{kg}$, reduces IOP 22.6\%, by indirectly lowering central venous pressure and venous pooling [11]. By contrast, succinylcholine increases IOP by direct contraction of the orbital smooth muscle, hindering aqueous outflow. Although topical application of a carbonic anhydrase inhibitor, a beta-blocker, an alpha-adrenoceptor agonist, or a prostaglandin analogue can be used to treat acute ocular hypertension [12], the benefit of these drugs must outweigh the risks of possible systemic adverse effects.

Factors other than anesthetic drugs can alter IOP. Manipulations that increase IOP include use of a laryngoscope, tracheal intubation, insertion of a laryngeal mask into the airway, hypoxia, hypercarbia, and patient position [3,13]. Laryngoscopic maneuvers and tracheal intubation are regarded as having the greatest effects on IOP [14]. Sympathetic stimulation during these procedures can alter patient hemodynamics and transiently elevate IOP. Hypoxia, hypercarbia, and coughing reflex during airway maneuvers may increase central venous pressure, resulting in an increase in IOP [13]. Patient position may also affect IOP; for example, in the Trendelenburg position with pneumoperitonium, the IOP may increase due to increased systemic blood pressure, partial pressure of end tidal carbon dioxide, or endobronchial pressure. In a steeper Trendelenburg position, greater than 30 degrees of bed tilting, optic hypoperfusion caused by the compartment syndrome of the optic nerve may lead to postoperative visual loss [2]. To manage the IOP surge, it is necessary to avoid venous pooling of the orbit by relieving the patient from the deep head down position, placing the orbit above the level of the heart [2,3]. Maintaining ocular perfusion pressure, calculated by subtracting IOP from mean arterial pressure, above the IOP can prevent hypoperfusion, which may lead to optic ischemia in a closed compartment [2]. The increases in IOP during intubation and emergence from anesthesia may be prevented by minimizing sympathetic stimulation and cough reflexes, and by maintaining patient hemodynamic variables within acceptable ranges. Patients who experience a sudden IOP surge should be kept in the reverse Trendelenburg position and treated with ophthalmic beta-blockers such as Timol eye drops $[2,8,14]$.

Portable tonometry is useful for monitoring IOP at the bedside. Tonopen is a light-weight portable device with a small diameter tip that has an accuracy equal to that of the Goldmann applanation tonometer, considered the gold standard for measuring IOP. Although measurements using the Tonopen and Goldmann applanation tonometers can differ more than 5 mmHg in approximately $7-8 \%$ of eyes, Tonopen measurements are within $\pm 3 \mathrm{mmHg}$ of Goldmann measurements in $>80 \%$ of eyes [15]. A statistical confidence indicator is shown on the screen as a percentage, thus indicating the accuracy of the measurement. We monitored IOP in our patient at regular intervals and during maneuvers such as intubation and position change to maintain IOP at constant levels. A portable tonometer can therefore provide point-of-care monitoring of IOP during general anesthesia.

In conclusion, patients with Posner-Schlossman syndrome can experience adverse events related to visual function upon undergoing non-ophthalmic surgery under general anesthesia. Serial measurements of perioperative IOP will provide clinical information useful for the safety of these patients. 


\section{References}

1. Kim TH, Kim JL, Kee C. Optic disc atrophy in patient with Posner-Schlossman syndrome. Korean J Ophthalmol 2012; $26: 473-7$.

2. Molloy BL. Implications for postoperative visual loss: steep trendelenburg position and effects on intraocular pressure. AANA J 2011; 79: 115-21.

3. Murphy DF. Anesthesia and intraocular pressure. Anesth Analg 1985; 64: 520-30.

4. Hunt K, Bajekal R, Calder I, Meacher R, Eliahoo J, Acheson JF. Changes in intraocular pressure in anesthetized prone patients. J Neurosurg Anesthesiol 2004; 16: 287-90.

5. Metwalli AR, Davis RG, Donovan JF. Visual impairment after laparoscopic donor nephrectomy. J Endourol 2004; 18: 888-90.

6. Mizrahi H, Hugkulstone CE, Vyakarnam P, Parker MC. Bilateral ischaemic optic neuropathy following laparoscopic proctocolectomy: a case report. Ann R Coll Surg Engl 2011; 93: e53-4.

7. Weber ED, Colyer MH, Lesser RL, Subramanian PS. Posterior ischemic optic neuropathy after minimally invasive prostatectomy. J Neuroophthalmol 2007; 27: 285-7.

8. Sator S, Wildling E, Schabernig C, Akramian J, Zulus E, Winkler M. Desflurane maintains intraocular pressure at an equivalent level to isoflurane and propofol during unstressed non-ophthalmic surgery. Br J Anaesth 1998; 80: 243-4.

9. Son YS, Oh SC, Chung KD, Kim KH, Yoon KJ. A comparison of the effects of propofol and sevoflurane anesthesias on intraocular pressure during laparoscopic hysterectomy. Korean J Anesthesiol 2005; 48: 10-4.

10. Sugata A, Hayashi H, Kawaguchi M, Hasuwa K, Nomura Y, Furuya H. Changes in intraocular pressure during prone spine surgery under propofol and sevoflurane anesthesia. J Neurosurg Anesthesiol 2012; 24: 152-6.

11. Jantzen JP, Hackett GH, Erdmann K, Earnshaw G. Effect of vecuronium on intraocular pressure. Br J Anaesth 1986; 58: 433-6.

12. Cheng JW, Cheng SW, Gao LD, Lu GC, Wei RL. Intraocular pressure-lowering effects of commonly used fixed-combination drugs with timolol: a systematic review and meta-analysis. PLoS One 2012; 7: e45079.

13. Kang KS, Bang KH, Ahn KR, Kwon JH, Lee JS. Effects of etomidate, thiopental sodium and propofol on intraocular pressure associated with tracheal intubation. Korean J Anesthesiol 2005; 48: 582-6.

14. Zimmerman AA, Funk KJ, Tidwell JL. Propofol and alfentanil prevent the increase in intraocular pressure caused by succinylcholine and endotracheal intubation during a rapid sequence induction of anesthesia. Anesth Analg 1996; 83: 814-7.

15. Bandyopadhyay M, Raychaudhuri A, Lahiri SK, Schwartz EC, Myatt M, Johnson GJ. Comparison of Goldmann applanation tonometry with the Tonopen for measuring intraocular pressure in a population-based glaucoma survey in rural West Bengal. Ophthalmic Epidemiol 2002; 9: 215-24. 